\title{
Twin "Fano-Snowflakes" over the Smallest Ring of Ternions
}

\author{
Metod SANIGA ${ }^{\dagger}$, Hans HAVLICEK ${ }^{\ddagger}$, Michel PLANAT $\S$ and Petr PRACNA \\ † Astronomical Institute, Slovak Academy of Sciences, \\ SK-05960 Tatranská Lomnica, Slovak Republic \\ E-mail: msaniga@astro.sk \\ URL: http://www.ta3.sk/ msaniga/ \\ $\ddagger$ Institut für Diskrete Mathematik und Geometrie, Technische Universität Wien, \\ Wiedner Hauptstraße 8-10, A-1040 Vienna, Austria \\ E-mail: havlicek@geometrie.tuwien.ac.at \\ $\S$ Institut FEMTO-ST/CNRS, MN2S, 32 Avenue de l'Observatoire, \\ F-25044 Besançon Cedex, France \\ E-mail: michel.planat@femto-st.fr
}

I J. Heyrovský Institute of Physical Chemistry, v.v.i., Academy of Sciences of the Czech Republic, Dolejškova 3, CZ-182 23 Prague 8, Czech Republic

E-mail: pracna@jh-inst.cas.cz

Received May 02, 2008, in final form May 30, 2008; Published online June 04, 2008

Original article is available at http://www.emis.de/journals/SIGMA/2008/050/

\begin{abstract}
Given a finite associative ring with unity, $R$, any free (left) cyclic submodule (FCS) generated by a unimodular $(n+1)$-tuple of elements of $R$ represents a point of the $n$-dimensional projective space over $R$. Suppose that $R$ also features FCSs generated by $(n+1)$-tuples that are not unimodular: what kind of geometry can be ascribed to such FCSs? Here, we (partially) answer this question for $n=2$ when $R$ is the (unique) non-commutative ring of order eight. The corresponding geometry is dubbed a "Fano-Snowflake" due to its diagrammatic appearance and the fact that it contains the Fano plane in its center. There exist, in fact, two such configurations - each being tied to either of the two maximal ideals of the ring - which have the Fano plane in common and can, therefore, be viewed as twins. Potential relevance of these noteworthy configurations to quantum information theory and stringy black holes is also outlined.
\end{abstract}

Key words: geometry over rings; non-commutative ring of order eight; Fano plane

2000 Mathematics Subject Classification: 51C05; 51Exx

Classical projective geometries, i.e., the ones where coordinates are elements of a (possibly skew) field, represent a venerable and well-developed branch of algebraic geometry. On the contrary, geometries over rings which are not fields, despite a quite abundant literature [1], still lie well outside the current mainstream research and there is still a lot to be done and discovered. Our interest in these geometries stems from the recent recognition of their importance for the field of quantum information theory (see, e.g., [2, 3, 4, 5, 6, 7] and references therein). Being motivated by this fact, we have had a detailed look at the fine structure of projective lines and, to a lesser extent, projective planes over a number of finite (both commutative and non-commutative) rings up to order 31. In doing so, we came across a number of interesting features [8] which have apparently not been treated so far by either mathematicians or physicists. This short note aims at acquainting the reader with, in our opinion, most fascinating of them. 
Let us consider an associative ring with unity $1(\neq 0), R$, and denote the left module on $n+1$ generators over $R$ by $R^{n+1}$. The set $R\left(r_{1}, r_{2}, \ldots, r_{n+1}\right)$, defined as follows

$$
R\left(r_{1}, r_{2}, \ldots, r_{n+1}\right):=\left\{\left(\alpha r_{1}, \alpha r_{2}, \ldots, \alpha r_{n+1}\right) \mid\left(r_{1}, r_{2}, \ldots, r_{n+1}\right) \in R^{n+1}, \alpha \in R\right\},
$$

is a left cyclic submodule of $R^{n+1}$. Any such submodule is called free if the mapping $\alpha \mapsto$ $\left(\alpha r_{1}, \alpha r_{2}, \ldots, \alpha r_{n+1}\right)$ is injective, i.e., if $\left(\alpha r_{1}, \alpha r_{2}, \ldots, \alpha r_{n+1}\right)$ are all distinct. Next, we shall call $\left(r_{1}, r_{2}, \ldots, r_{n+1}\right) \in R^{n+1}$ unimodular if there exist elements $x_{1}, x_{2}, \ldots, x_{n+1}$ in $R$ such that

$$
r_{1} x_{1}+r_{2} x_{2}+\cdots+r_{n+1} x_{n+1}=1 .
$$

It is a very well-known fact (see, e.g., $[9,10,11])$ that if $\left(r_{1}, r_{2}, \ldots, r_{n+1}\right)$ is unimodular, then $R\left(r_{1}, r_{2}, \ldots, r_{n+1}\right)$ is free; any such free cyclic submodule represents a point of the $n$-dimensional projective space defined over $R$ [10]. The converse statement, however, is not generally true. That is, there exist rings which also give rise to free cyclic submodules featuring exclusively non-unimodular $(n+1)$-tuples, i.e., free cyclic submodules that cannot be associated with any point of the corresponding projective space. The first case when this happens is the unique non-commutative ring of order eight, $R_{\diamond}$, i.e., the ring isomorphic to the one of upper (or lower) triangular two-by-two matrices over the Galois field of two elements. Let us therefore have a detailed look at this case.

To this end in view, we first introduce the standard matrix representation of $R_{\diamond}[12,13]$,

$$
R_{\diamond} \equiv\left\{\left(\begin{array}{cc}
a & b \\
0 & c
\end{array}\right) \mid a, b, c \in G F(2)\right\},
$$

from where it is readily seen that the ring contains two maximal (two-sided) ideals,

$$
I_{1}=\left\{\left(\begin{array}{ll}
0 & b \\
0 & c
\end{array}\right) \mid b, c \in G F(2)\right\}
$$

and

$$
I_{2}=\left\{\left(\begin{array}{cc}
a & b \\
0 & 0
\end{array}\right) \mid a, b \in G F(2)\right\},
$$

which give rise to a non-trivial (two-sided) Jacobson radical $J$,

$$
J=I_{1} \cap I_{2}=\left\{\left(\begin{array}{ll}
0 & b \\
0 & 0
\end{array}\right) \mid b \in G F(2)\right\} .
$$

As for our further purposes it will be more convenient to work with numbers than matrices, we shall relabel the elements of $R_{\diamond}$ as follows

$$
\begin{aligned}
& 0 \equiv\left(\begin{array}{cc}
0 & 0 \\
0 & 0
\end{array}\right), \quad 1 \equiv\left(\begin{array}{cc}
1 & 0 \\
0 & 1
\end{array}\right), \quad 2 \equiv\left(\begin{array}{cc}
1 & 1 \\
0 & 1
\end{array}\right), \quad 3 \equiv\left(\begin{array}{ll}
1 & 1 \\
0 & 0
\end{array}\right) \\
& 4 \equiv\left(\begin{array}{cc}
0 & 0 \\
0 & 1
\end{array}\right), \quad 5 \equiv\left(\begin{array}{cc}
1 & 0 \\
0 & 0
\end{array}\right), \quad 6 \equiv\left(\begin{array}{cc}
0 & 1 \\
0 & 0
\end{array}\right), \quad 7 \equiv\left(\begin{array}{cc}
0 & 1 \\
0 & 1
\end{array}\right) \text {. }
\end{aligned}
$$

The addition and multiplication in the ring is that of matrices over $G F(2)$, which in our compact notation reads as shown in Table 1 (adopted, with a slight notational difference, from [14]). From the multiplication table (as well as from expressions (1)) it follows that, apart from unity, there is only one more invertible element, 2 , and that the zero-divisors are of two kinds: nilpotent (0 and 6$)$ and idempotent $(3,4,5$, and 7$)$. The two maximal ideals now acquire the form

$$
I_{1}:=\{0,4,6,7\} \quad \text { and } \quad I_{2}:=\{0,3,5,6\} \text {, }
$$


Table 1. Addition (left) and multiplication (right) in $R_{\diamond}$.

\begin{tabular}{||c|cccccccc||}
\hline \hline+ & 0 & 1 & 2 & 3 & 4 & 5 & 6 & 7 \\
\hline 0 & 0 & 1 & 2 & 3 & 4 & 5 & 6 & 7 \\
1 & 1 & 0 & 6 & 7 & 5 & 4 & 2 & 3 \\
2 & 2 & 6 & 0 & 4 & 3 & 7 & 1 & 5 \\
3 & 3 & 7 & 4 & 0 & 2 & 6 & 5 & 1 \\
4 & 4 & 5 & 3 & 2 & 0 & 1 & 7 & 6 \\
5 & 5 & 4 & 7 & 6 & 1 & 0 & 3 & 2 \\
6 & 6 & 2 & 1 & 5 & 7 & 3 & 0 & 4 \\
7 & 7 & 3 & 5 & 1 & 6 & 2 & 4 & 0 \\
\hline \hline
\end{tabular}

\begin{tabular}{||c|cccccccc||}
\hline \hline$\times$ & 0 & 1 & 2 & 3 & 4 & 5 & 6 & 7 \\
\hline 0 & 0 & 0 & 0 & 0 & 0 & 0 & 0 & 0 \\
1 & 0 & 1 & 2 & 3 & 4 & 5 & 6 & 7 \\
2 & 0 & 2 & 1 & 3 & 7 & 5 & 6 & 4 \\
3 & 0 & 3 & 5 & 3 & 6 & 5 & 6 & 0 \\
4 & 0 & 4 & 4 & 0 & 4 & 0 & 0 & 4 \\
5 & 0 & 5 & 3 & 3 & 0 & 5 & 6 & 6 \\
6 & 0 & 6 & 6 & 0 & 6 & 0 & 0 & 6 \\
7 & 0 & 7 & 7 & 0 & 7 & 0 & 0 & 7 \\
\hline \hline
\end{tabular}

and the Jacobson radical reads,

$$
J=I_{1} \cap I_{2}=\{0,6\} .
$$

Confining to the case of $n=2$, we will now demonstrate that there exist triples of elements $\left(r_{1}, r_{2}, r_{3}\right) \in R_{\diamond}^{3}$ which are not unimodular and yet they generate free cyclic submodules.

It is a straightforward task to verify that such triples are tied uniquely to the first maximal ideal $\left(I_{1}\right)$; here, we shall list them implicitly together with the corresponding free cyclic submodules:

$$
\begin{aligned}
R_{\diamond}(4,6,7) & =R_{\diamond}(7,6,4) \\
& =\{(0,0,0),(4,6,7),(7,6,4),(6,6,0),(4,0,4),(0,6,6),(6,0,6),(7,0,7)\}, \\
R_{\diamond}(4,7,6) & =R_{\diamond}(7,4,6) \\
& =\{(0,0,0),(4,7,6),(7,4,6),(6,0,6),(4,4,0),(0,6,6),(6,6,0),(7,7,0)\}, \\
R_{\diamond}(6,4,7) & =R_{\diamond}(6,7,4) \\
& =\{(0,0,0),(6,4,7),(6,7,4),(6,6,0),(0,4,4),(6,0,6),(0,6,6),(0,7,7)\}, \\
R_{\diamond}(4,4,7) & =R_{\diamond}(7,7,4) \\
& =\{(0,0,0),(4,4,7),(7,7,4),(6,6,0),(4,4,4),(0,0,6),(6,6,6),(7,7,7)\}, \\
R_{\diamond}(4,7,4) & =R_{\diamond}(7,4,7) \\
& =\{(0,0,0),(4,7,4),(7,4,7),(6,0,6),(4,4,4),(0,6,0),(6,6,6),(7,7,7)\}, \\
R_{\diamond}(7,4,4) & =R_{\diamond}(4,7,7) \\
& =\{(0,0,0),(7,4,4),(4,7,7),(0,6,6),(4,4,4),(6,0,0),(6,6,6),(7,7,7)\}, \\
R_{\diamond}(4,4,6) & =R_{\diamond}(7,7,6) \\
& =\{(0,0,0),(4,4,6),(7,7,6),(6,6,6),(4,4,0),(0,0,6),(6,6,0),(7,7,0)\}, \\
R_{\diamond}(4,6,4) & =R_{\diamond}(7,6,7) \\
& =\{(0,0,0),(4,6,4),(7,6,7),(6,6,6),(4,0,4),(0,6,0),(6,0,6),(7,0,7)\}, \\
R_{\diamond}(6,4,4) & =R_{\diamond}(6,7,7) \\
& =\{(0,0,0),(6,4,4),(6,7,7),(6,6,6),(0,4,4),(6,0,0),(0,6,6),(0,7,7)\}, \\
R_{\diamond}(6,6,7) & =R_{\diamond}(6,6,4) \\
& =\{(0,0,0),(6,6,7),(6,6,4),(6,6,0),(0,0,4),(6,6,6),(0,0,6),(0,0,7)\}, \\
R_{\diamond}(6,7,6) & =R_{\diamond}(6,4,6) \\
& =\{(0,0,0),(6,7,6),(6,4,6),(6,0,6),(0,4,0),(6,6,6),(0,6,0),(0,7,0)\}, \\
R_{\diamond}(7,6,6) & =R_{\diamond}(4,6,6) \\
&
\end{aligned}
$$




$$
\begin{aligned}
& =\{(0,0,0),(7,6,6),(4,6,6),(0,6,6),(4,0,0),(6,6,6),(6,0,0),(7,0,0)\}, \\
R_{\diamond}(0,6,7) & =R_{\diamond}(0,6,4) \\
& =\{(0,0,0),(0,6,7),(0,6,4),(0,6,0),(0,0,4),(0,6,6),(0,0,6),(0,0,7)\}, \\
R_{\diamond}(0,7,6) & =R_{\diamond}(0,4,6) \\
& =\{(0,0,0),(0,7,6),(0,4,6),(0,0,6),(0,4,0),(0,6,6),(0,6,0),(0,7,0)\}, \\
R_{\diamond}(0,4,7) & =R_{\diamond}(0,7,4) \\
& =\{(0,0,0),(0,4,7),(0,7,4),(0,6,0),(0,4,4),(0,0,6),(0,6,6),(0,7,7)\}, \\
R_{\diamond}(6,0,7) & =R_{\diamond}(6,0,4) \\
& =\{(0,0,0),(6,0,7),(6,0,4),(6,0,0),(0,0,4),(6,0,6),(0,0,6),(0,0,7)\}, \\
R_{\diamond}(7,0,6) & =R_{\diamond}(4,0,6) \\
& =\{(0,0,0),(7,0,6),(4,0,6),(0,0,6),(4,0,0),(6,0,6),(6,0,0),(7,0,0)\}, \\
R_{\diamond}(4,0,7) & =R_{\diamond}(7,0,4) \\
& =\{(0,0,0),(4,0,7),(7,0,4),(6,0,0),(4,0,4),(0,0,6),(6,0,6),(7,0,7)\}, \\
R_{\diamond}(6,7,0) & =R_{\diamond}(6,4,0) \\
& =\{(0,0,0),(6,7,0),(6,4,0),(6,0,0),(0,4,0),(6,6,0),(0,6,0),(0,7,0)\}, \\
R_{\diamond}(7,6,0) & =R_{\diamond}(4,6,0) \\
& =\{(0,0,0),(7,6,0),(4,6,0),(0,6,0),(4,0,0),(6,6,0),(6,0,0),(7,0,0)\}, \\
R_{\diamond}(4,7,0) & =R_{\diamond}(7,4,0) \\
& =\{(0,0,0),(4,7,0),(7,4,0),(6,0,0),(4,4,0),(0,6,0),(6,6,0),(7,7,0)\} .
\end{aligned}
$$

We find altogether 42 non-unimodular triples of elements of $R_{\diamond}^{3}$ generating 21 distinct free left cyclic submodules which in their entirety comprise all 64 triples of elements formed from $I_{1}$. These submodules are, as illustrated in Fig. 1, very intricately "interwoven" with each other as:

- the triple $(0,0,0)$ (not shown in the figure) is the common meet of all of them;

- each of the seven triples $(6,0,0),(0,6,0),(0,0,6),(6,6,0),(6,0,6),(0,6,6),(6,6,6)$ (represented by big circles in the figure) shares nine of them;

- each of the 14 triples $(4,0,0),(0,4,0),(0,0,4),(4,4,0),(4,0,4),(0,4,4),(4,4,4),(7,0,0)$, $(0,7,0),(0,0,7),(7,7,0),(7,0,7),(0,7,7),(7,7,7)$ (medium-size circles) has three of them in common; and

- each of the remaining 42 triples (small circles) lies on a unique submodule.

It is obvious from Fig. 1 that the seven triples of the set listed in the second item above can be viewed as the points of the smallest projective plane, the Fano plane. As these triples are seen to form specific three-member subsets each of which defines a unique aggregate of submodules of cardinality three, and there are just seven such aggregates (distinguished in Fig. 1 by different colors), these aggregates of submodules can be regarded as the lines of this plane. This is a truly remarkable property because the entries of the seven triples come from $J$, which is a ring of order two without unity, i.e., the ring not isomorphic to the Galois field of two elements. Turning now to the second maximal ideal $\left(I_{2}\right)$, we find that we cannot create from it any non-unimodular triple generating free left cyclic submodules. If, however, we switch to right cyclic submodules instead, we do find the configuration completely identical in its shape to that shown in Fig. 1; moreover, as $J$, by its very definition, belongs to the both maximal ideals, the two configurations share the same Fano plane and, so, can be regarded as twin (or dual to each other) "Fano-Snowflakes".

The Fano plane occurs in algebraic geometry and geometric algebra in various disguises $[15,16]$, and is perhaps most recognized as a "gadget" completely describing the algebra structure 


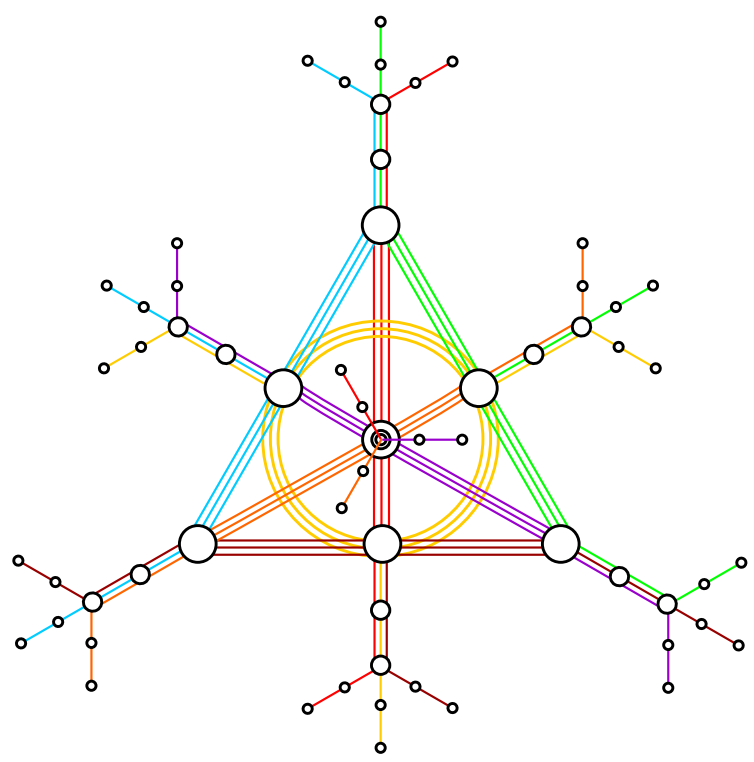

Figure 1. The "Fano-Snowflake" - a diagrammatic illustration of a very intricate relation between the 21 free left cyclic submodules generated by non-unimodular triples of $R_{\diamond}^{3}$ (represented here by the circles of different sizes as explained in the text). As the $(0,0,0)$ triple is not shown, each submodule is represented by seven circles (three big, two medium-sized and two small) lying on a common segmented/broken line. The colors were chosen in such a way to make (the lines of) the Fano plane sitting in the middle of the configuration readily discernible. There are seven "protrusions", or "extensions", of the Fano plane emanating from its points, each giving rise to three more ramifications. (The "protrusion/extension" emanating from the point at the very center is illustrated as going perpendicularly out of the sheet of page so that only its three ramifications can be seen properly.) On a side note, any of the seven sets of three submodules represented by the same color stands for an $n=1$ case.

of the octonions [17]. It is, therefore, not surprising that this plane has also found numerous applications in physics (coding and information theory, network-switching, etc.). In this context it is especially worth mentioning recently-discovered relations between stringy black holes and quantum information theory. We have in mind intriguing mathematical coincidences between black-hole solutions in string theory and quantum entanglement in certain finite-dimensional Hilbert spaces, where some symmetry structures relevant to string theories are encoded into the incidence structure of the Fano plane. In particular, different types of so-called $E_{7}$-symmetric black-hole solutions can neatly be classified in terms of different types of entangled quantum states attached to the points/lines of the Fano plane and the black hole entropy formula based on the Fano plane yields an entanglement measure of seven qubits [18, 19, 20, 21]. It may well happen that the above-described generalized/extended Fano configurations, and a variety of their relatives to be discovered over many higher-order non-commutative rings, provide a geometric link between more involved stringy black holes and more complex forms of quantum entanglement featured by multipartite quantum systems. It is already the next case in the hierarchy, viz. the ring of ternions over the field of three elements, which deserves a detailed inspection. Here, in analogy with the Fano case, we find a "snowflake" centered around the projective plane of order three (see Fig. 2) ${ }^{1}$. This projective plane has a noteworthy link to a few sporadic simple groups [22], including the largest one, the Monster group [23]. And the Monster is intimately connected with another wide class of 3D black holes, so-called BTZ black holes [24], as the logarithm of the dimension of one of its representations yields the entropy

\footnotetext{
${ }^{1}$ Based on these two cases, we surmise that the corresponding configuration always features $P G(2, q)$ as the core geometry, irrespectively of the order $q$ of the base field of the ring of ternions.
} 


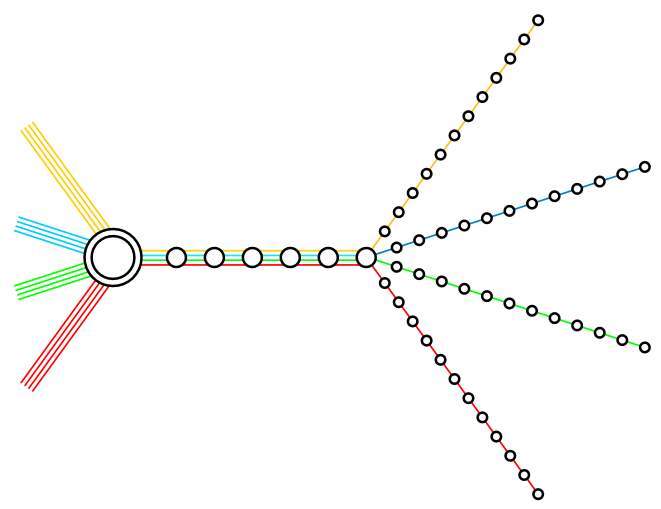

Figure 2. A sketchy illustration - in the symbols and notation of Fig. 1 - of a generic part of the "Snowflake" created by free left cyclic submodules generated by non-unimodular triples of the ring of ternions over $G F(3)$; the double-circle stands, on the one hand, for two distinct triples of the Jacobson radical of the ring and, on the other hand, for a single point of the underlying projective plane of order three.

of such a black hole [25]. It is, therefore, our hope that this paper will stir the equal interest of both mathematicians and theoretical physicists: the former into a systematic treatment of free cyclic submodules generated by non-unimodular vectors, the latter into a serious search for possible applications of the associated geometrical configurations.

\section{Acknowledgements}

The work was supported by the VEGA grant agency projects Nos. 6070 and 7012, the CNRSSAV Project No. 20246 "Projective and Related Geometries for Quantum Information" and by the Action Austria-Slovakia project No. 58s2 "Finite Geometries Behind Hilbert Spaces".

\section{References}

[1] Törner G., Veldkamp F.D., Literature on geometry over rings, J. Geom. 42 (1991), 180-200.

[2] Planat M., Saniga M., Kibler M.R., Quantum entanglement and projective ring geometry, SIGMA 2 (2006), 066, 14 pages, quant-ph/0605239.

[3] Saniga M., Planat M., Pracna P., Havlicek H., The Veldkamp space of two-qubits, SIGMA 3 (2007), 075, 7 pages, arXiv:0704.0495.

[4] Havlicek H., Saniga M., Projective ring line of a specific qudit, J. Phys. A: Math. Theor. 40 (2007), F943F952, arXiv:0708.4333.

[5] Planat M., Baboin A.-C., Qudits of composite dimension, mutually unbiased bases and projective ring geometry, J. Phys. A: Math. Theor. 40 (2007), F1005-F1012, arXiv:0709.2623.

[6] Havlicek H., Saniga M., Projective ring line of an arbitrary single qudit, J. Phys. A: Math. Theor. 41 (2008), 015302, 12 pages, arXiv:0710.0941.

[7] Saniga M., Planat M., Pracna P., Projective ring line encompassing two-qubits, Theor. and Math. Phys. 155 (2008), 463-473, quant-ph/0611063.

[8] Saniga M., A fine structure of finite projective ring lines, an invited talk given at the workshop on Prolegomena for Quantum Computing (November 21-22, 2007, Besançon, France), slides of the talk are available at http://hal.archives-ouvertes.fr/hal-00199008.

[9] Veldkamp F.D., Projective planes over rings of stable rang 2, Geom. Dedicata 11 (1981), 285-308.

[10] Veldkamp F.D., Geometry over rings, in Handbook of Incidence Geometry, Editor F. Buekenhout, Elsevier, Amsterdam, 1995, 1033-1084. 
[11] Herzer A., Chain geometries, in Handbook of Incidence Geometry, Editor F. Buekenhout, Elsevier, Amsterdam, 1995, 781-842.

[12] Benz W., Zur Umkehrung von Matrizen im Bereich der Ternionen, Mitt. Math. Ges. Hamburg 10 (1979), 509-512.

[13] Lex W., Poneleit V., Weinert H.J., Über die Einzigkeit der Ternionenalgebra und linksalternative Algebren kleinen Ranges, Acta Math. Acad. Sci. Hungar. 35 (1980), 129-138.

[14] Nöbauer C., The book of the rings - part I (2000), pages 65 and 76, available online from http://www . algebra. uni-linz.ac.at/ noebsi/pub/rings.ps.

[15] Polster B., A geometrical picture book, Springer, New York, 1998, Chapter 5.

[16] Brown E., The many names of (7,3,1), Math. Mag. 75 (2002), 83-94.

[17] Baez J., The octonions, Bull. Amer. Math. Soc. (N.S.) 39 (2002), 145-205, math.RA/0105155.

[18] Lévay P., Stringy black holes and the geometry of entanglement, Phys. Rev. D 74 (2006), 024030, 16 pages, hep-th/0603136.

[19] Lévay P., Strings, black holes, the tripartite entanglement of seven qubits and the Fano plane, Phys. Rev. D 75 (2007), 024024, 19 pages, hep-th/0610314.

[20] Duff M.J., Ferrara S., $E_{6}$ and the bipartite entanglement of three qutrits, Phys. Rev. D 76 (2007), 124023, 7 pages, arXiv:0704.0507.

[21] Duff M.J., Ferrara S., $E_{7}$ and the tripartite entanglement of seven qubits, Phys. Rev. D 76 (2007), 025018, 7 pages, quant-ph/0609227.

[22] Conway J.H., Elkies N.D., Martin J.L., The Mathieu group $M_{12}$ and its pseudogroup extension $M_{13}$, Experiment. Math. 15 (2006), 223-236, math.GR/0508630.

[23] Conway J.H., Simons C.S., 26 implies the bimonster, J. Algebra 235 (2001), 805-814.

[24] Bañados M., Teitelboim C., Zanelli J., The black hole in three dimensional space time, Phys. Rev. Lett. 69 (1992), 1849-1851, hep-th/9204099.

[25] Witten E., Three-dimensional gravity revisited, arXiv:0706.3359. 